\title{
The Challenges of Teaching Finite Element Analysis in the Undergraduate Curriculum
}

Nader G. Zamani, Ph.D. P.Eng.

\author{
University of Windsor, Canada, zamani@uwindsor.ca
}

\begin{abstract}
The widespread availability of commercial FEA
software in the academic institutions and pressure from industry to include it in the modern undergraduate curriculum has resulted in unique challenges in teaching the topic to undergraduate students who may or may not be adequately prepared to handle the subject. The present expository paper shares the author's experiences in delivering the FEA subject to university students both at the undergraduate and the graduate levels. The most common pitfalls for the understanding of the tool, particularly by the undergraduate students are highlighted.

Keywords-undergraduate curriculum, finite element analysis, commercial software, mechanics.
\end{abstract}

Digital Object Identifier (DOI):

http://dx.doi.org/10.18687/LACCEI2016.1.1.028

ISBN: 978-0-9822896-9-3

ISSN: 2414-6390

$14^{\text {th }}$ LACCEI International Multi-Conference for Engineering, Education, and Technology: "Engineering Innovations for Global Sustainability", 20-22 July 2016, San José, Costa Rica. 


\title{
The Challenges of Teaching Finite Element Analysis in the Undergraduate Curriculum
}

\author{
Nader G. Zamani, Ph.D. P.Eng. \\ University of Windsor, Canada, zamani@uwindsor.ca
}

\begin{abstract}
The widespread availability of commercial FEA software in the academic institutions and pressure from industry to include it in the modern undergraduate curriculum has resulted in unique challenges in teaching the topic to undergraduate students who may or may not be adequately prepared to handle the subject. The present expository paper shares the author's experiences in delivering the FEA subject to university students both at the undergraduate and the graduate levels. The most common pitfalls for the understanding of the tool, particularly by the undergraduate students are highlighted.

Keywords - undergraduate curriculum, finite element analysis, commercial software, mechanics.
\end{abstract}

\section{INTRODUCTION}

Although the fundamentals of the topic of the finite element analysis date back to the last century, the modern and practical aspects of it are relatively new and traced back to the 1960s. This coincided with the development of several commercial finite element software [1], [2], and [3] to name a few. During the 70s and 80s, significant research activities on the solid mechanics side was undertaken and by the early 90 s the use of FEA was prevalent in industry and the graduate programs in universities. In general, graduate courses (intermediate and advanced) were made available for interested students which provided them with a good understanding of the subject beyond the use of a software. Due to the consolidation of FEA software packages, and the widespread availability of powerful and inexpensive personal computers, the finite element topic has gradually crept into the undergraduate engineering curriculum. Just about every engineering undergraduate engineering program has a course on FEA at the third or fourth year level. In fact, in some schools, it is introduced in the freshman year.

The inclusion of finite elements in the undergraduate curriculum has resulted in many positive contributions but at the same time created unique challenges in properly teaching it. At the undergraduate level, the appropriate prerequisites for taking, understanding, and utilizing FEA are listed below. These courses are, engineering mechanics (both statics and dynamics), strength of materials, engineering materials, introductory stress analysis, and ideally, a basic course in mechanical vibrations. Essentially, these prerequisites are completed by the end of the third year. Therefore, the optimal point for introducing FEA to the undergraduates is in the final term of the third year.

The major factors that complicate the issue are twofold. The FEA software have become so "user friendly" that a typical

Digital Object Identifier (DOI): http://dx.doi.org/10.18687/LACCEI2016.1.1.028 ISBN: 978-0-9822896-9-3

ISSN: 2414-6390

14 ${ }^{\text {th }}$ LACCEI International Multi-Conference for Engineering, Education, and Technology: "Engineering Innovations for Global Sustainability", 20-22 July 2016, Costa Rica undergraduate student assumes that the results are correct and makes no attempt to question the validity of the output. In particular, to some students, if the contour plot is generated and the "colors look good", the results must be correct. Furthermore, there has been a major push by the software companies to suppress (or downplay) the availability of the "theoretical manuals" to the extent that the users (undergraduates) have no recourse in knowing how the results have been arrived at. Needless to say that the "devil is in the details". It may be true that a typical undergraduate may not consult the theoretical manual but this information may not be even available to the course instructors and even researchers. In the remaining part of this paper, the author's experience with a variety of problems on which the students may have conceptual difficulties are discussed. Although the FEA presentation in this paper is using the CATIA v5 program [4], it could have been done with any other commercial software.

\section{THE RESTRAINTS AND LOADS}

Students often do not realize that that the restraints and loads applied as boundary conditions are mathematical idealization of real situation and simply try to approximate the physical condition. This conceptual difficulty in FEA, stems from earlier courses such as engineering mechanics and strength of materials. At that stage, the instructors are so involved in teaching the students the mechanisms of how to arrive at the final solution that often ignore such issues.

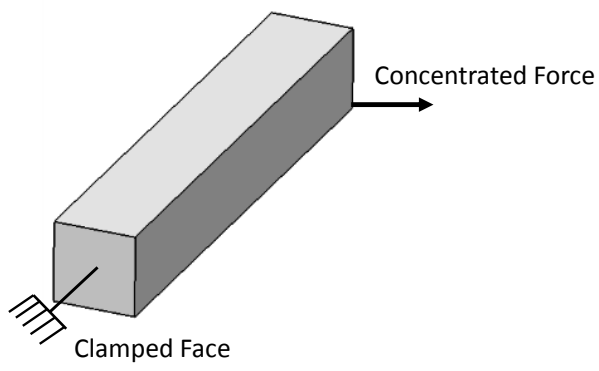

Fig. 1 to be emphasized that neither the clamped face, nor concentrated loads can physically be achieved

The students should be reminded that the physical restraints and loads are the results of multi-part interaction. Furthermore, that a concentrated force is the result of a pressure acting over an extremely small area. It is worth referring to a simple situation such as depicted in Fig. 1 and to emphasize that neither the clamped face, nor the concentrated force are physically realizable. 


\section{THE MESH SIZE DILLEMA}

In order to make the current FEA packages "look" user friendly, the number of warning messages is kept at minimum. This is partly the reason that the students usually look for the "deflection" plot without paying much attention to the actual numbers. Clearly, ignoring the element size, can dramatically affect the stress values and to a lesser extent, the deflection values. In order to convince the students of the significance of the mesh size, a simple cantilever beam problem can be used. To be more specific, a 1x1x10 inch steel beam whose end face is clamped, and the top face is subjected to a pressure of $50 \mathrm{psi}$. is considered. This simple geometry is used to avoid confounding the issue.
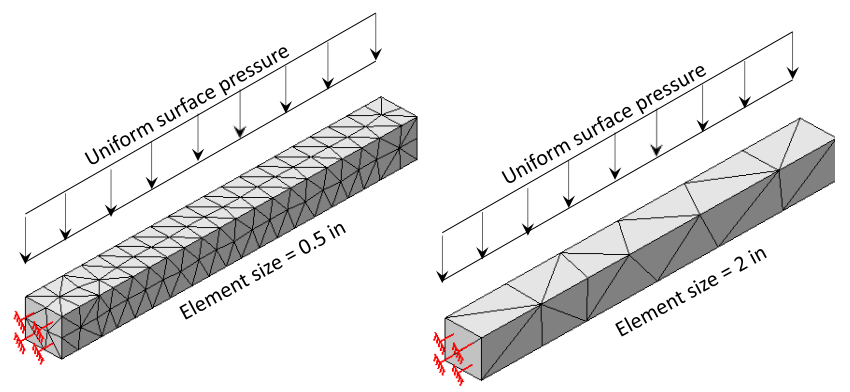

Fig. 2 Element size effect on deflection and stress

The two different discretization of the cantilever problem are shown in Fig. 2, where the coarse mesh is 2 in. whereas the fine mesh is $0.5 \mathrm{in}$. The results of using linear tetrahedral elements with the coarse and fine mesh are described in Table I. The quantities compared are the maximum deflection and the maximum von Mises stress.

TABLE I

EFFECT OF ELEMENT SIZE

\begin{tabular}{|c|c|c|}
\hline $\begin{array}{c}\text { Element } \\
\text { size } \\
\text { (inches) }\end{array}$ & $\begin{array}{c}\text { Maximum } \\
\text { Deflection } \\
\text { (inches) }\end{array}$ & $\begin{array}{c}\text { Maximum von } \\
\text { Mises } \\
\text { Stress (psi) }\end{array}$ \\
\hline 2.0 & 0.0056 & 3410 \\
\hline 0.5 & 0.0155 & 8200 \\
\hline
\end{tabular}

A typical student, having seen such a comparison, arrives at the conclusion that a smaller element size results in a more reliable solution. Therefore, they select an extremely small size which can bring the computer to stall trying to complete the job.

The students should be convinced through a good example that although small elements are preferred, their effectiveness depends on where they are deployed. These elements are to be used in locations where the stress contours are tightly packed together. A good demonstration problem is a $0.5 \times 4 \times 8$ rectangular plate made of steel with a central hole of radius 0.25 (all dimensions are in inches). The plate is under tension with a load of 1000 psi. The plate thickness is assumed to be $0.5 \mathrm{in}$.
The setup is shown in Fig 3, where the nominal mesh for both parts is the same size, however, one of the parts has a modest local mesh refinement in the critical area.
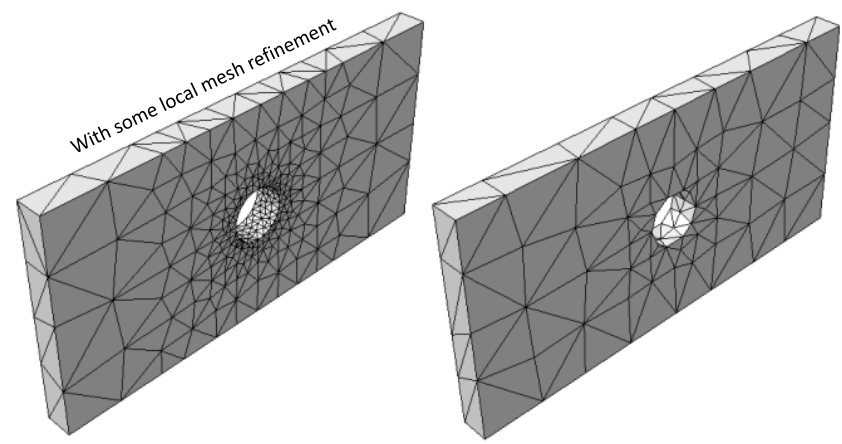

Fig. 3 The effect of local mesh refinement

Hopefully, the results described in Table II convinces the students that not all elements need to be made small, but only those in critical areas. Furthermore, it warns the students that the deflections are not as sensitive to the mesh size as the stresses are. This is a point that is often neglected by the beginners.

TABLE II

EFFECT OF LOCAL MESH REFINEMENT

\begin{tabular}{|c|c|c|}
\hline & $\begin{array}{c}\text { Max Deflection } \\
\text { (inches) }\end{array}$ & $\begin{array}{c}\text { Max von } \\
\text { Mises } \\
\text { Stress (psi) }\end{array}$ \\
\hline No local refinement & $2.94 \mathrm{E}-4$ & 1820 \\
\hline $\begin{array}{c}\text { With local } \\
\text { refinement }\end{array}$ & $2.97 \mathrm{E}-4$ & 2780 \\
\hline
\end{tabular}

\section{STATIC VS DYNAMIC}

It is the author's opinion that the confusion of the distinction between static and dynamic by an average student, has nothing to do with the subject of finite elements. It has to do with the impression that static analysis does not involve time whereas dynamic analysis involves time variation. The best approach to rectify this misunderstanding is to explain the phenomenon in terms of the mass-spring system under a ramped load as shown in Fig. 4.

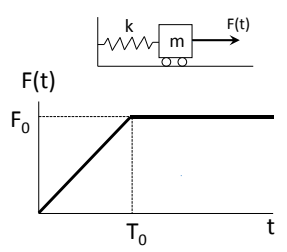

Fig. 4 The mass-spring system under a damped load 
For the sake of concreteness, let us select the following numerical values. Assume that , $\mathrm{m}=10 \mathrm{~kg}, \mathrm{k}=10000 \mathrm{~N} / \mathrm{m}$, and $\mathrm{F}_{0}=100 \mathrm{~N}$. The point of this example is to explain the significance of the parameter $\mathrm{T}_{0}$ which explains the difference between a static and dynamic response. Keep in mind that $\mathrm{T}_{0}$ represents how fast the ramp load rises to the final constant value of $\mathrm{F}_{0}=100 \mathrm{~N}$. The students already know that the natural frequency of free vibration of the system is given by $\omega=$ $\sqrt{k / m}=31.623 \mathrm{rad} / \mathrm{s}$. This frequency corresponds to a period $T=\frac{2 \pi}{\omega}=0.2 \mathrm{~s}$. Two values of $\mathrm{T}_{0}$ are now examined. In the first case $T_{0}=6 T$ whereas in the second case $T_{0}=0.5 T$. The graphs of the mass displacement as function of time are displayed in Fig. 5.

The graphs convince the students that the same physical problem can behave in two different ways depending how fast the load is applied. It is worth pointing out to the students that in the case of $T_{0}=6 T$, one can use the specified time, find the magnitude of the load at that time, and solve the static equation $K x=F$. This approach is not valid when dealing with the case $T_{0}=0.5 T$. The example just presented also emphasizes the importance of knowing the natural frequencies of the structure in order to decide on the solution strategy.
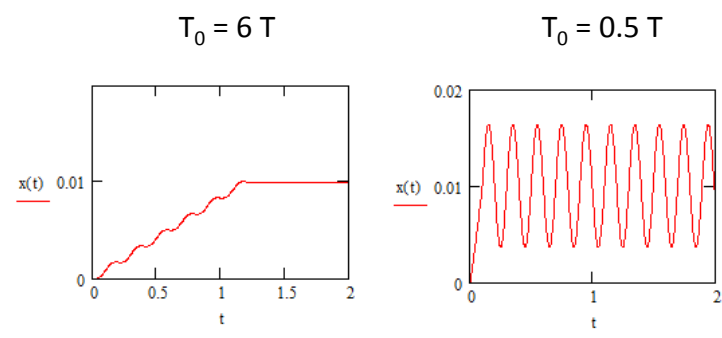

Fig. 5, mass displacement as a function of time

\section{NATURAL FREQUENCIES}

The previous section emphasized the role of the natural frequency in the behavior of the system response. The concept of natural frequencies is not well understood by some mechanical engineering students. The topic of FEA is the natural point in the curriculum to clarify this issue. Finite elements is a versatile tool to demonstrate the different scenarios involving natural frequencies. Perhaps an example involving resonance clarifies the idea. Figure 6, displays a simply supported beam with a motor placed at the mid-span. The beam is made of steel with approximate dimensions $2.5 \mathrm{x}$ $3.2 \times 25.5$ inches.

The first ten natural frequencies of the beam are calculated with CATIA and are displayed in Fig. 7. The associated first four modes are also displayed in Fig. 8. A substantial amount of time must be devoted to the interpretation of the frequency values and the corresponding mode shapes by the instructors.
The students should be warned that although tens of thousands of natural frequencies can be calculated in FEA, this involves substantial CPU time and are of no practical use. Depending on the problem, at most handful of frequencies can ever be employed and are sufficiently accurate to have any significance. They should also be warned that the magnitude of the displacements and stresses are meaningless. It is the relative displacement that is of importance.

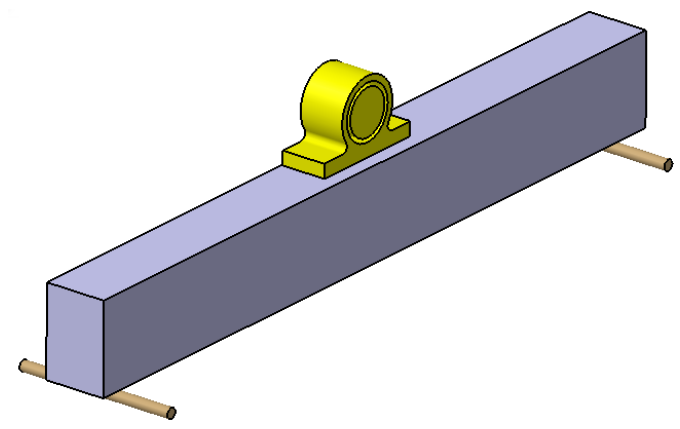

Fig. 6, simply supported beam

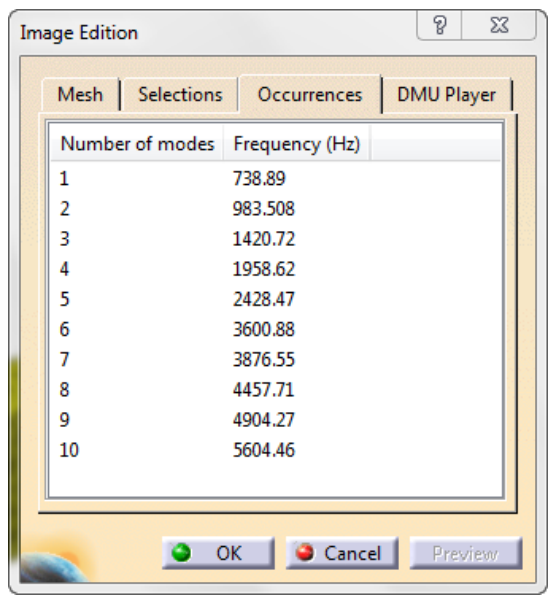

Fig. 7, the first ten natural frequencies of the beam

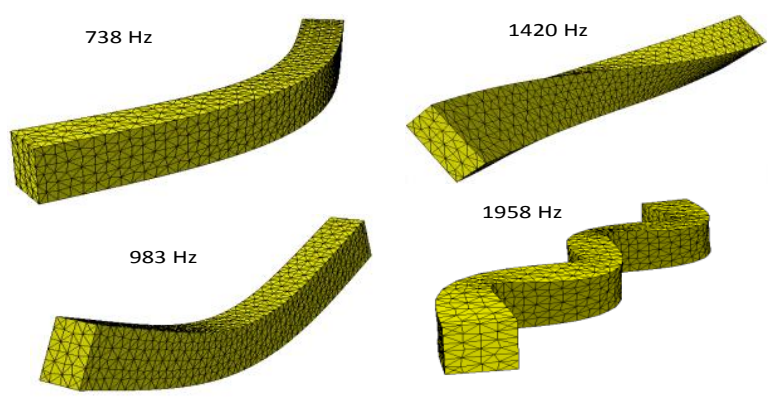

Fig. 8, the lowest four modes 


\section{SYMMETRY CONSIDERATIONS}

The concept of reducing an FEA domain due to symmetry considerations is rather difficult to comprehend by the majority of the undergraduate students. Part of the difficulty may be that they view the part geometry in isolation and if they recognize planes of symmetry (geometric symmetry), they immediately conclude that there is also finite element symmetry. Through simple models, they have to be convinced that the "loads" and "restraints" also play a role in this matter. Three scenarios such as the ones displayed in Fig. 9 can serve the purpose.

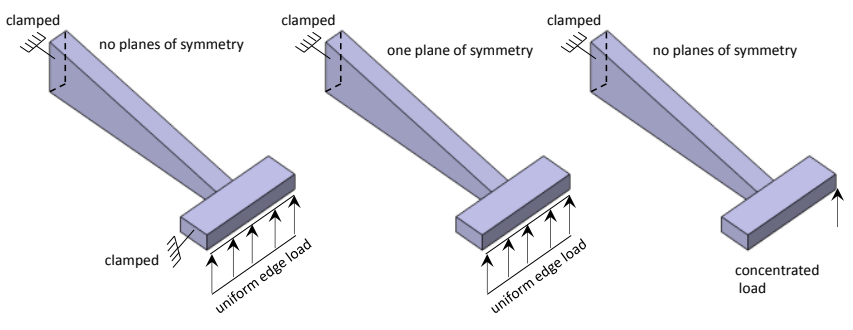

Fig. 9, the role of geometry, load, and restraint in determining the number of planes of symmetry

Due to the importance of the application of symmetry in finite elements, it is worth mentioning other fields of mechanical engineering where this issue arises. At this point in their education, the students should be familiar with the fundamentals of heat transfer. The geometry in Fig. 10 can be viewed as a concrete slab with longitudinal cavities carrying a heated fluid (say air) of a constant temperature. The external boundaries of the slab are subject to free convection.

As long as the cavities are reasonably away from the two side ends, one can investigate a single cell (level 1 symmetry), or even further reduction (level 2 symmetry), both shown in the same figure. This reduction is traditionally referred to as "cyclic symmetry".

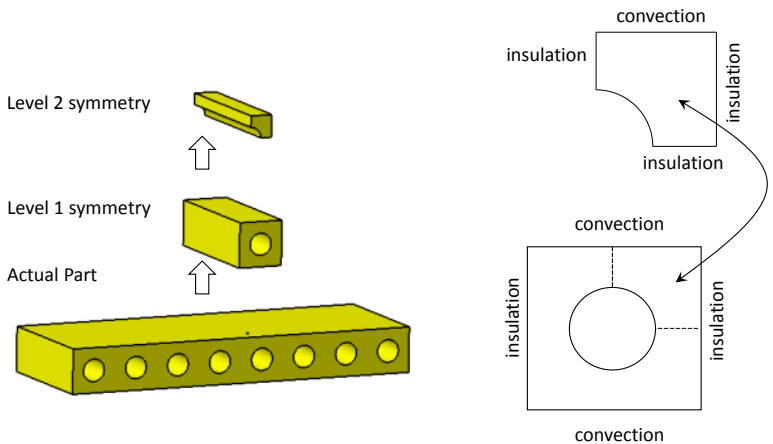

Fig. 10, cyclic symmetry in heat transfer applications
Irrespective of these explanations, a typical student is generally not easily convinced about the advantages of employing the symmetry conditions. The most common argument by the student is, "why do have to complicate life, if the entire problem can be modelled, ignoring any symmetry considerations?". Needless to say, on the surface, this sounds as a valid question.

The students should be reminded that in principle, smaller elements lead to more reliable results. By using symmetry, one can substantially reduce the size of the part under consideration as shown in Fig 10, and therefore a large number of elements can cover a smaller domain. The students should also be warned that the reducing the model by symmetry considerations, in certain cases such as natural frequency or buckling load calculations can suppress certain deformation modes that may be critical to the interpretation of the results. Figure 11, is a reminder that in the natural frequency calculations of a simply supported beam, if symmetry is used, all the anti-symmetric modes of vibration are suppressed.

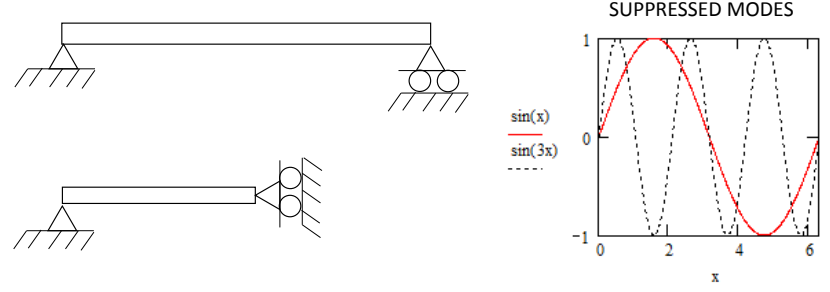

Fig. 11, mode suppression effect

\section{ELEMENT TYPES}

In all likelihood, the students are initially exposed to solid elements and therefore they have the tendency of discretizing every part with such elements. In fact, it is the case that solid elements produce the most reliable (and complete) results but only if they are sufficiently small in size. The three types of structural elements (in the simplest form), namely, "Solid", "Shell" and "Beam" are displayed in Fig. 12. The associated degrees of freedom are also displayed in the figure.
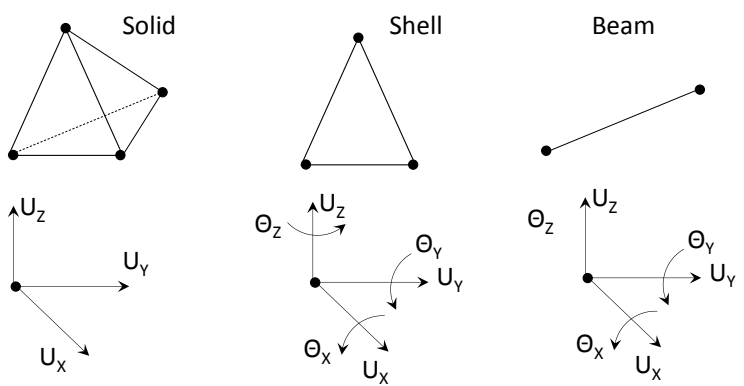

Fig. 12, Solid, Shell, Beam elements and their degrees of freedom

$14^{\text {th }}$ LACCEI International Multi-Conference for Engineering, Education, and Technology: "Engineering Innovations for 
It takes a great deal of effort and a large number of examples to convince a student of the suitability of using a specific type of element for given part. Some suggestions are provided in Fig. 13. The general explanation can rely on the relative dimensions in different directions. For example, if the dimensions of the part in two directions are much smaller than the third direction, the "beam" element may be appropriate. In situations where one particular direction is substantially smaller than the other two, "shell" elements are perhaps appropriate. The above two statements are not strictly correct, but are more reassuring to an undergraduate student.

They students should also be cautioned that in real engineering problems requiring FEA, all three type of elements may be needed to generate realistic results. When dealing with such cases, one should be very careful in interpreting the stress distribution at the junction of two different type of elements.

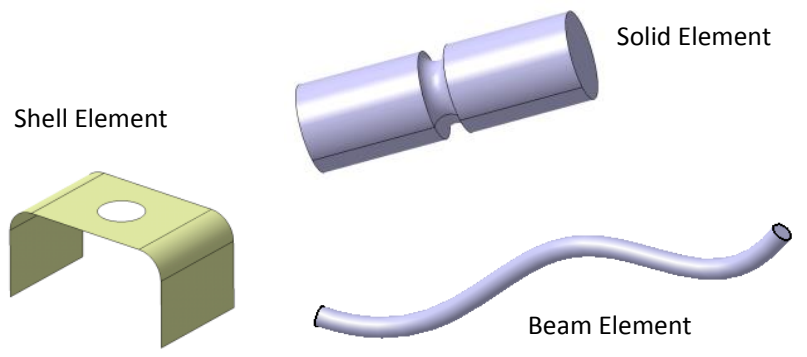

Fig. 13, Solid, Shell, Beam elements and their degrees of freedom

It is worth spending some time on discussing these elements individually and highlighting their features. For example, there are different beam formulations such as "Bernoulli" and "Kirchhoff" types. If the beam is slender, the shear deformation may be negligible and therefore the Bernoulli beams are appropriate. On the other hand, for "short-fat" beams, Kirchhoff formulation is more appropriate. Furthermore, not all beam elements are capable of simulating a cable behavior as they cables do not resist compression whereas beams do. Ordinarily, there are certain features in the software that can be activated to turn a beam into a cable element.

Similarly, there are different types of shell elements. In the case of very thin shells, the shear deformation can be safely ignored, whereas shear can have a major effect in the case of thick shells. The junction between shells of different thicknesses can display erroneous stress distribution. This comment also applies to the junction of beams with different cross sections. To get detailed information at the junction, one will have to use solid elements in that location.

The symmetry considerations discussed in section $\mathrm{V}$ are also applicable to the beam and shell elements. Since these elements have rotational degrees of freedom, they cause a confusion among the students.

To alleviate this issue, table III shown below can be used to enforce the appropriate restraints. For example, if the edge of a shell element lies in the YZ symmetry plane, one needs to apply the restraints $U_{X}=0, \Theta_{Y}=0, \Theta_{Z}=0$.

TABLE III

TABLE FOR RESTRAINTS UNDER DIFFERENT SYMMETRY PLANES

\begin{tabular}{|c|c|c|c|c|c|c|}
\hline PLANE & $\mathrm{U}_{\mathrm{X}}$ & $\mathrm{U}_{\mathrm{Y}}$ & $\mathrm{U}_{\mathrm{Z}}$ & $\Theta_{\mathrm{X}}$ & $\Theta_{\mathrm{Y}}$ & $\Theta_{\mathrm{Z}}$ \\
\hline $\mathrm{YZ}$ & zero & free & free & free & zero & zero \\
\hline $\mathrm{XZ}$ & free & zero & free & zero & free & zero \\
\hline $\mathrm{XY}$ & free & free & zero & zero & zero & free \\
\hline
\end{tabular}

The reader may be familiar with SAE Mini-Baja competition [5], [6] in which engineering schools from across the world have the opportunity to participate (see Fig 14). One of the aspect of this competition is to design a frame which has the structural integrity based on the SAE specifications. Ordinarily, because of the complexity of the geometry, the participating teams use a commercial FEA software to investigate their design. It is very common that they use solid elements to model components (such as tubes) in order to predict the stresses. This will frequently lead to poor results as it cannot be discretized with sufficiently small solid elements. The students' reasoning for this decision is based on convenience and the lack of familiarity with the beam and shell elements.

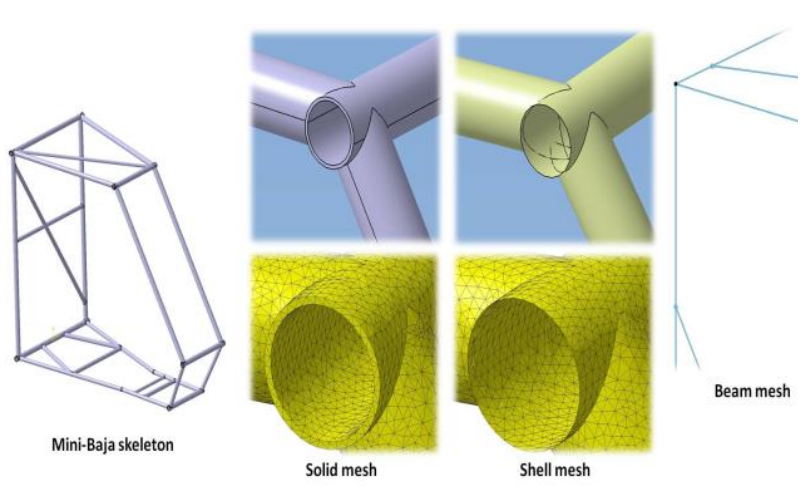

Fig. 14, Mini-Baja frame modeled with solid, shell, and beam elements

\section{RIGID BODY MOTION AND SMALL PIVOTS}

One of the issues facing the undergraduate students using the finite element tool is to be able to identify and resolve error messages generated by the software caused by rigid body motion. The actual message generated, varies among the FEA software but they all point to a matrix singularity or small pivots. Generally speaking, the students resort to the most convenient solution which could be "randomly" clamping (or enforcing zero displacements) until the software runs. Naturally, they are pleased that some results are generated and

$14^{\text {th }}$ LACCEI International Multi-Conference for Engineering, Education, and Technology: "Engineering Innovations for Global Sustainability”, 20-22 July 2016, San José, Costa Rica. 
therefore, having the false sense of security that the output can be interpreted. In the case of the CATIA v5 commercial software, the dialogue box for such errors is displayed in Fig. 15. Note that the message contains key words such as "Singularity detected", "Pivot too small", and a possible reason for such a deficiency. In most software, some displacement values are available for which are all extremely large and meaningless. The students are encouraged to plot the deformation (which are rigid body motion) in order to possibly arrive at the root cause of the software failure.

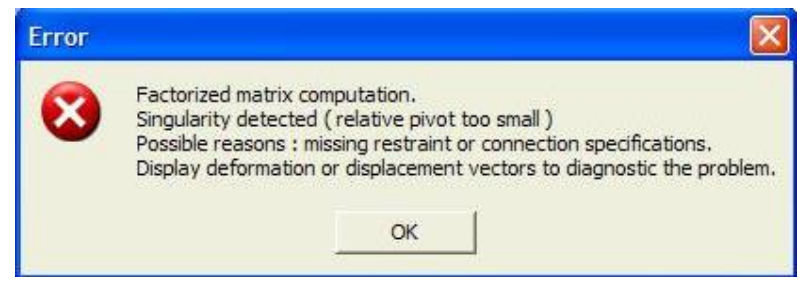

Fig. 15, CATIA v5 error message for "rigid body" mode

Perhaps the best way to illustrate these issues is in terms of a lumped two degree of freedom system as shown in Fig. 16. On the top of the figure, the time varying variables along with the masses are displayed. The equation of motion describing this system is given in (1).

$$
\left[\begin{array}{cc}
m & 0 \\
0 & m
\end{array}\right]\left[\begin{array}{l}
\ddot{U}_{1}(t) \\
\ddot{U}_{2}(t)
\end{array}\right]+\left[\begin{array}{cc}
k & -k \\
-k & k
\end{array}\right]\left[\begin{array}{l}
U_{1}(t) \\
U_{2}(t)
\end{array}\right]=\left[\begin{array}{l}
F_{1}(t) \\
F_{2}(t)
\end{array}\right]
$$

Once the initial conditions of the two masses are prescribed, the system can be integrated in time and the solution $U_{1}(t), U_{2}(t)$ is obtained. Now consider the static situation shown in the bottom of Fig. 16. There, the forces are constant and therefore, the system is under equilibrium. This implies that $U_{1}, U_{2}$ are independent of time and (1) above simplifies to (2), described below.

$$
\left[\begin{array}{cc}
k & -k \\
-k & k
\end{array}\right]\left[\begin{array}{l}
U_{1} \\
U_{2}
\end{array}\right]=\left[\begin{array}{c}
F \\
-F
\end{array}\right]
$$

Note that the stiffness matrix is singular (zero pivot) and system cannot be solved uniquely for the displacements. Physically speaking, the location of the system can be anywhere along the horizontal line and it still remains in equilibrium.

Notice that this is consistent with the "Error" message content, namely "missing restraint". If one of the masses is fixed (ie zero displacement), position of the other mass is uniquely determined from (2).
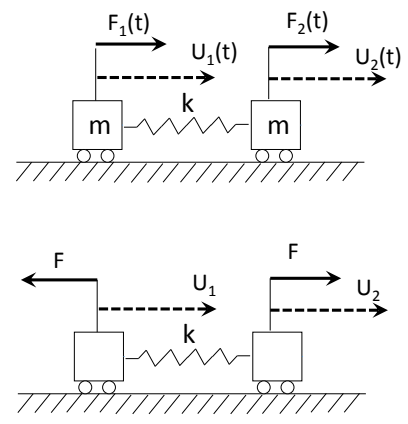

Fig. 16, rigid body motion dynamic vs static case

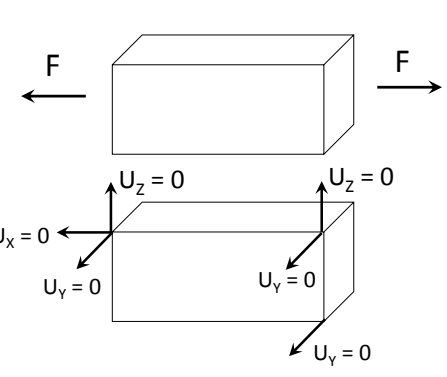

(a)

(b)

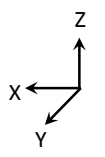

Fig. 17, the 1-2-3 rule to eliminate rigid body motion

The situation is naturally more complicated in the case of distributed system such as a part to be analyzed, but the underlying principle is the same. As a concrete example, consider a block which is under static equilibrium as shown in Fig 17(a). In order to analyze this structure, one should apply certain restraints with the provision that the rigid body motion is eliminated but they contribute to no artificial stresses in the block. The minimal restraints at three locations are shown in Fig. 17 (b), also known as the 1-2-3 rule which does not lead to any fictitious stresses but eliminates the rigid body motion. The common question by the students is, "how does one decide on which points to fix and which degrees of freedom?". This is a perfectly legitimate question but unfortunately it requires experience, thinking, and sometimes trial and error. In the event that the students are using CATIA v5, there is a restraint icon named "Isostatic" which in principle applies the above rule automatically. Unfortunately it is frequently misused by the students leading to erroneous results. This type of restraint is needed to simulate the spring-back in metal forming simulations. 


\section{NONLINEAR GEOMETRY}

The type of the FEA problems that an undergraduate mechanical engineering student is ordinarily is facing with is of linear type. This means, infinitesimal deformations and strains. Furthermore, it is assumed that that the Hooke's law prevails. Because of the superposition principle, the student can impose any load (force and/or pressure), and the software will generate the corresponding output (displacement and stress). Notwithstanding the magnitude of the displacement and stress, the deformed shape and the contours of stress may look reasonable but the values may be totally meaningless. For example, a part that is roughly $1 \mathrm{~m}$ in size, can have displacements of $1 \mathrm{Km}$. The software does not check for material nonlinearities (such as yielding) and therefore some students do not question the validity of the results. Quite frequently, they ask the instructor whether their answer is correct or not.

It is helpful to remind the students about the implications of the superposition principle. This can be done in the context of a simple linear spring of stiffness $\mathrm{k}$, with a fixed end, while loaded with a concentrated force $F$ at the other end. The displacement of the loaded end is given by linear expression $K U=F$. increasing the value of $\mathrm{F}$ by a factor of "c" leads to increasing the value of $U$ by the same factor, without violating the "linear physics" behind the model. The moral of the story is to warn the students not apply "irresponsible" loads as the software will not complain.

\section{MATERIAL PROPERTIES AND FAILURE}

As it was pointed out earlier, at the undergraduate level, the students are not heavily exposed to the intricate issues in material modelling. In all likelihood, the bulk of the topics that they are exposed to deal with linear, homogenous, and isotropic martials. It is tempting to ignore the isotropy condition and try to model parts made out of wood which is orthotropic. Naturally, the FEA results cannot be trusted and must be handled with care. It is important to point out to the students that orthotropic material can behave elastically, so modeling such parts with FEA is not necessarily difficult. However, they should consult the software documentation to know what material information is needed and whether it is readily available.

In the case linear isotropic material, the three parameters, Young's modulus, Poisson's ratio, and mass density are what is needed. For wood however, both the Young's modulus and Poisson's ratio are direction dependent. The author's experience is that at least few lectures must be devoted to the review of engineering materials topic. The students should particularly review the properties of metals, wood, polymers, concrete, and soil.
A universal question by the undergraduates (and others) is "Does my part break?". This is a complicated and non-trivial question and they should be asked in return, "What do you mean by break?". To many students, the term "break" refers to a catastrophic event (failure) where part literally breaks into two pieces. Clearly, this is one particular mode of failure but there many other modes. Other modes of failure can be excessive deflection, yielding, failure due to fatigue, and rupture. Although such topics are ordinarily covered in a machine design course, typical third year students lack the maturity and in-depth understanding of the topic. Typically, they concentrate on yielding. At this level, it is probably best to emphasize the design based on preventing "yield". This can readily be done as the default stress is the von Mises stress in most commercial FEA software. The students should be constantly reminded that the von Mises failure criterion is primarily intended for ductile failure and should not be applied to brittle materials such as glass, ceramics, composites, and concrete.

\section{ANALYSIS OF ASSEMBLY VS PARTS}

An undergraduate course in finite elements usually involves the analysis of a single part. However, to fully appreciate the importance of FEA, it is highly recommended to let the students experiment with the analysis of simple assemblies. To be more specific, let us take the assembly shown in Fig. 18 which is consisting of two parts. It is assumed that there is a tight fit, ie the diameter of the shaft and the hole are exactly the same. Furthermore, it is assumed that the shaft and the hole are perfectly bonded (or glued) together.

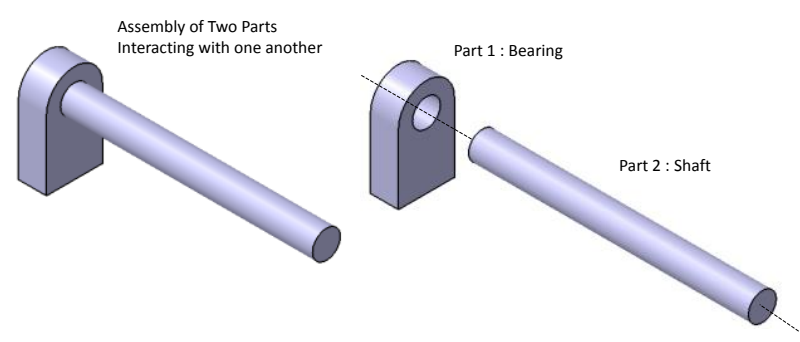

Fig. 18, the Shaft-Bearing assembly

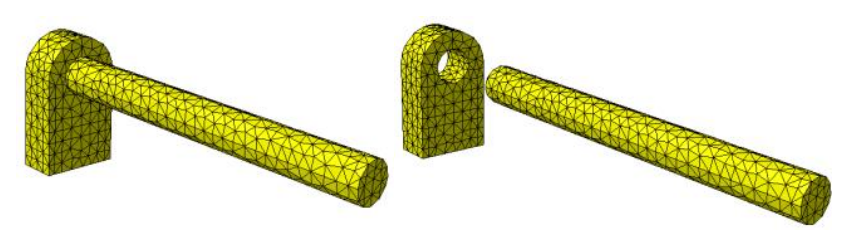

Fig. 19, the mesh in the assembled and unassembled configurations

14 ${ }^{\text {th }}$ LACCEI International Multi-Conference for Engineering, Education, and Technology: "Engineering Innovations for Global Sustainability", 20-22 July 2016, San José, Costa Rica. 
For the sake of illustration, the two meshes are shown both in the assembled and unassembled configurations, as shown in Fig. 19.

The restraint and the load are described next. The bottom face of the bearing is completely clamped and the free end of the shaft is subjected to a distributed downward force. Without an exception, when the students model the assembly, they do not realize that the two parts as they stand, do not interact with each other.

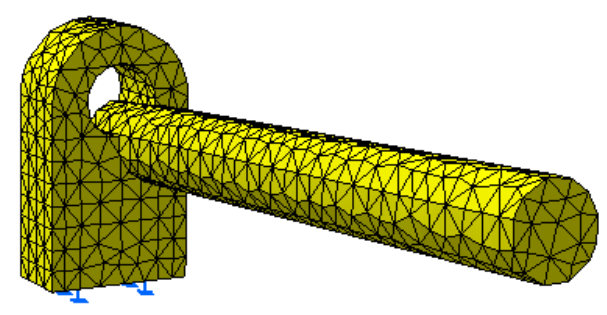

Fig. 20, the shaft moves as a rigid body cutting through the bearing

Another way to phrase this is that, the two parts "do not talk to each other". The consequence of this observation is that the shaft begins to move as a rigid body and cuts through the bearing as shown in Fig. 20. The core of the complexity in analyzing an assembly is modelling (defining) the interaction between various parts. In the present problem, it was indicated that the two parts are perfectly bonded (glued). In CATIA v5 this can be achieved by first defining an "Analysis Connection" between the two parts, and then declaring it as a "Fastened Connection". Once, the interaction is defined, upon running the software, the deformation is shown in Fig. 21 is displayed which is what one expects.

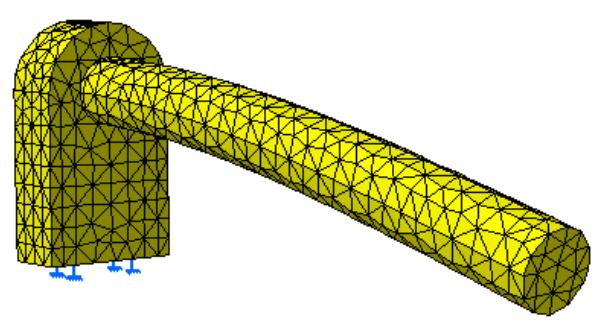

Fig. 21, the deflected shape after defining the interaction

The students should be cautioned that not all interactions are of "Fastened Connection" type. For example, in reality there is a finite gap between the shaft and the hole (specified tolerance) and in that situation, a "Contact Connection" is appropriate. It is important for them to consult the software user's manual to know exactly what is available and their limitations.

\section{ISSUES WITH INFINITE STRESSES}

A problem that arises frequently is the issue of applying a load as a concentrated force vs pressure. In Fig. 22, a clamped bar (at its left face) is subjected to a tensile load as shown. The load is modeled in two different ways. The first approach is to divide the magnitude of the load by the number of nodes on that face, and apply this value to every single node. Clearly this is statically equivalent to the intended load.

The deformations result is displayed in the same figure (the second one from the top). One can see that there are major problems with this plot. The students may not see the issue at the outset but a good explanation by the instructor brings it to their attention. Note the unusual deformation pattern at the corners of the end face. The consequence of this unacceptable deformation is huge fictitious stresses that are developed at the corners. Generally speaking the usage of concentrated forces in solid elements results in unrealistic large stresses under the load which can mask the entire picture. The students should think twice before using concentrated forces in solid elements.

The proper way to model this tensile load is to apply it as pressure, as shown in the bottom of Fig.22. There are no unusual deformations at the corners, and the stress is uniform throughout the cross section as expected.

The students are surprised to know that the infinite stress scenario can also arise in other circumstances where there are no concentrated forces. A good example for demonstration is
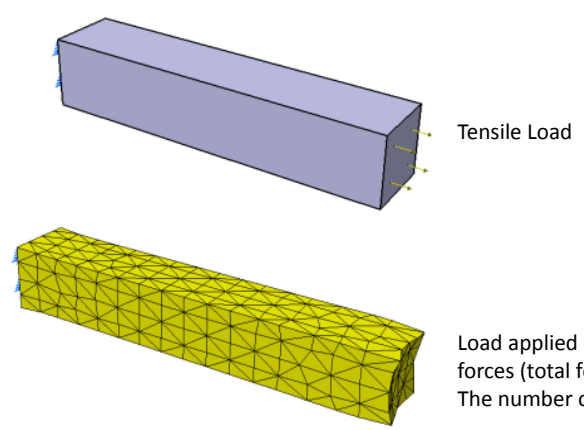

Load applied as concentrated forces (total force divided by The number of nodes on the face)

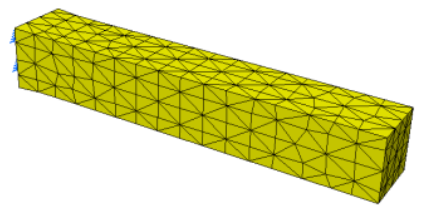

Load applied as pressure (total force divided by area)

Fig. 22, fictitious deformation developed at the corners of the face

the linear elastic analysis of a part with a reentrant corner (such as a sharp crack). The situation is displayed in Fig. 23. When the applied load results in the opening of the sharp crack, the exact stress value at the crack tip is infinite. This is the basis of "Linear Elastic Fracture Mechanics". 


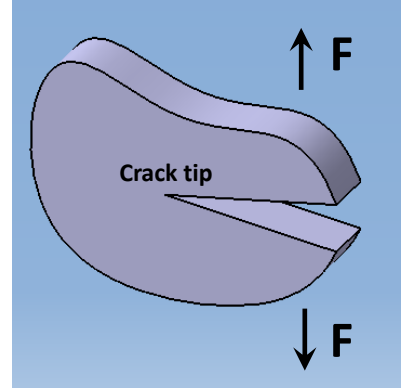

Fig. 23, infinite stress at a reentrant corner (crack tip)

Presenting the above problem to the students is a good "teachable moment" to discuss the significance of using "fillets" in critical locations with the reentrant corner characteristics. Use of generous fillet radius should be encouraged as long as it does not interfere with the functionality of the part. Another point to emphasize is that although the theoretical stress distribution may be infinite, in practice, the material yields in such locations and the infinite value is never achieved. This statement assumes that one is not concerned about fatigue failure. The situations is considerably more difficult in fatigue failure.

\section{APPLYING LOAD AND RESTRAINT AT ARBITRARY LOCATIONS WITH NO FEATURES}

The proliferation of CAE/CAD/FEA in the industrial and academic sectors has lead the development of user friendly software which are mostly parametric, feature based packages. To make this statement more explicit, suppose that a student/user wants to apply a pressure load on a circular region on the top face of the block shown in Fig. 24.

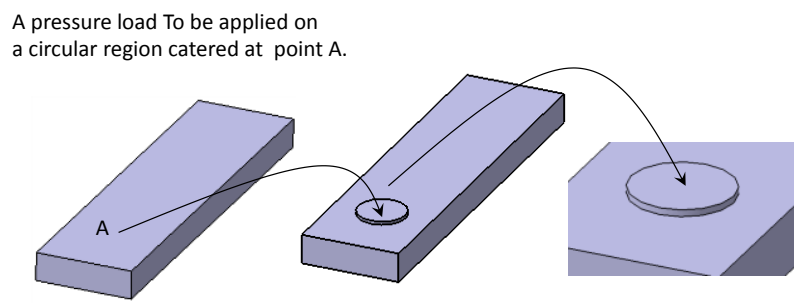

Fig. 24, pressure to be applied on a circle centered at A

In majority of modern CAE software, if the surface on which the point " $\mathrm{A}$ " is located is selected, the pressure is applied on the entire surface. To a typical student, a way to get around is to create a feature around the point " $\mathrm{A}$ " such as the circular stand displayed in the figure, and then apply the pressure on the created circular surface. This strategy in referred to as an "embossment" in the CAD terminology. Although this may be reasonable in certain problems, it could be problematic in general. For example, stress concentration may be resulting due to the height of the padded/extruded embossment.
There are specific approaches to avoid this issue which are software dependent and the students should be made aware of them. In the CATIA v5 program (that our students are using), the process involves creating a circular surface on the top face which obviously has no height, and 'sewing" the created surface to the top face of the block. This creates a feature on which the pressure load can be applied to. This process in CATIA v5 is described in Fig. 25.
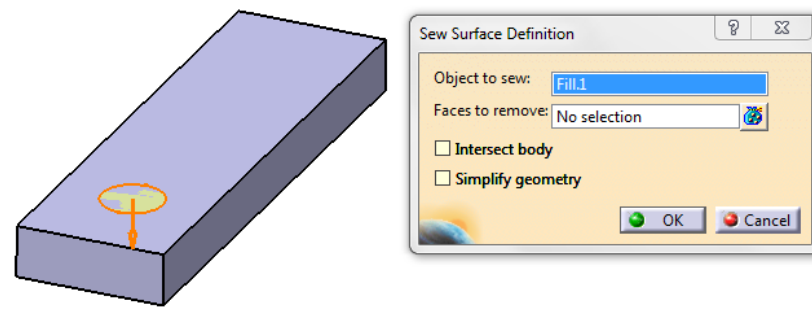

Fig. 25, sewing the surface to the top face

When the part is meshed, the software recognizes that the presence of the "Sewed" surface as shown in Fig 26.
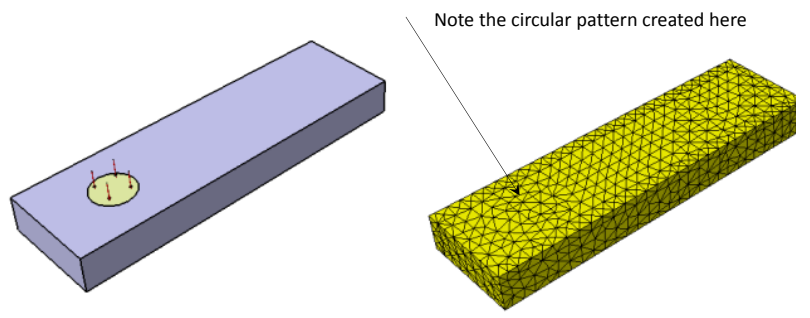

Fig. 26, pressure can be applied on the feature

\section{STRUGGLING WITH THE VIRTUAL PARTS}

In most commercial software, there are capabilities which are based on RBE2 and RBE3 from the NASTRAN program developed in the early 60s. The "virtual part" option in CATIA v5 is a variation of the above elements with certain modifications. The toolbar associated with "virtual parts" is shown in Fig. 27.

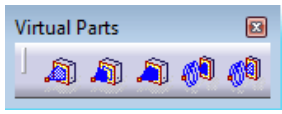

Fig. 27, the virtual part tool bar in CATIA v5

Undoubtedly, in the analysis module, the virtual part capability is the "least understood and the most abused" feature by the students. This statement also applies to more seasoned finite element analysts. The core of the issue is the lack of proper documentation in the software and disregarding the limitations of the feature. The usage and description of virtual 
element is usually in terms of the displacement degrees of freedom but the complications are in terms of specifying the load on the virtual part. To be more concrete, consider the line shown in Fig. 28 which is meshed with the ten beam elements [7]. A "smooth" virtual part is created with the support being the line and the handler point being at two different locations, namely point " $A$ " directly above the centroid and point " $B$ " above the right end of the line, as shown in Fig. 28.
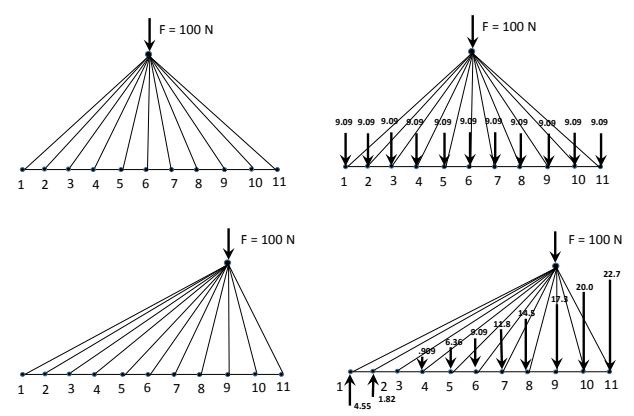

Fig. 28, load transfer process for a "smooth virtual" part

By glancing through the load distribution displayed in Fig. 28, it becomes evident that the non-trivial formulation is playing a significant role. The uniform distribution of the load as shown is normally what the students expect. This however applies only when the load goes through the support's centroid. The figure is adopted from a training document for the RBE3 in the NASTRAN program [8]. The CATIA formulation for "smooth virtual" part seems to be an adaptation of such an element.

\section{BEAM ELEMENT OFFSET}

It was pointed out earlier that it takes some effort to convince the students of the need to use different types of elements in a single model (a hybrid model). Although the message may be relayed to the students and appreciated by them, implementing them in a meaningful way can be a challenge. This is frequently observed in using beam elements.

The setting in Fig. 29 is the situation where beam-solid or beam-shell elements are needed. The students in general generate a surface (or a solid) and on the surface draw lines which are later meshed with beam elements. However, if the offset of the beam it not specified, the beams are acting as shown in the bottom of the Fig. 29. In this manner, they act as "stiffeners" instead of a foundation support. Clearly, the deflection results and therefore stresses are not accurately representing the design intent.
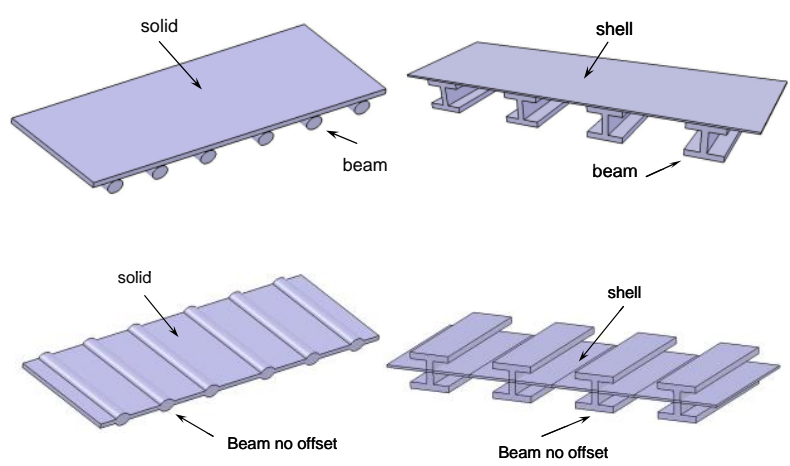

Fig. 29, the effect of beam offset in a hybrid model [9]

The offset feature in the majority of the commercial finite element packages are not well explained and appear in an obscure "check box" or "radio button" that needs to be activated. Needless to say that students do not enthusiastically look for the feature under discussion.

\section{CONCLUDING REMARKS}

Undoubtedly, the finite element analysis plays a critical role in the modern mechanical engineering curriculum. The author has attempted to outline the common challenges by the students to learn and effectively use this valuable tool. The pedagogical points raised are not exhaustive but hopefully alerts and assists the instructor in such a course to maximize the learning opportunities by the students.

\section{REFERENCES}

[1] MacNeal-Schwendler Corporation, MSC NASTRAN General purpose Finite Element program, Newport Beach California, 2015

[2] ANSYS Inc., Cannonsburg, Pennsylvania, 2015.

[3] ABAQUS CAE, Simulia, Dassault Systemes Corporation. Providence, Rhode Island, 2015

[4] N.G. Zamani, CATIA v5 FEA Tutorials, Release 21, SDC Publications, Mission Kansas, 2012.

[5] SAE mini Baja, "The Mini-Baja Competition Guidelines", Society of Automotive Engineers, 2015.

[6] B. Shahabi, Master's Thesis, "A Structural Investigation to Develop Guidelines for the Finite Element Analysis of the Mini-Baja Vehicle", University of Windsor, 2011.

[7] CATIA v5, Documentation Manual (Elfini) Dassault Systemes Corporation. Providence, Rhode Island, 2015

[8] MSC Nastran, Training Manual, "RBEs and MPCs in MSC NATRAN", Newport Beach California, 2015.

[9] N. G. Zamani, N. Gharib, P.N. Kaloni, "Two Case Studies for the Effect of Beam Offset in Beam Calculations", International Journal of Engineering Education, pp 87-97, Volume 37, Number 2, 2009.

14 ${ }^{\text {th }}$ LACCEI International Multi-Conference for Engineering, Education, and Technology: "Engineering Innovations for 